\title{
Tipping the splicing balance to kill cancer cells
}

Mutations in genes encoding splicing factors are commonly found in samples from patients with several haematological cancers, as well as some solid tumours. A new paper in Nature Medicine describes a novel, orally available splicing modulator that preferentially kills cancer cells with mutations in splicing factors in culture and in mouse models.

Tumour cells with mutations in the splicing machinery are particularly sensitive to splicing modulation. Although the most common cancerassociated mutations in splicing factors cause aberrant splicing through seemingly different mechanisms, all of them either reduce the production of canonical mRNA that is suitable for translation or induce new transcripts.

Pladienolide reduces the production of mature mRNA by binding to and modulating the splicing factor $3 \mathrm{~b}$ (SF3b) subcomplex, which is characterised by the presence of SF3B1. "There were several splice modulators, but none of them showed the proprieties we wanted for targeting these newly identified splicing factor mutations," explains corresponding author Silvia Buonamici. Through an iterative medicinal chemistry approach starting with pladienolide, they identified a compound that competes with

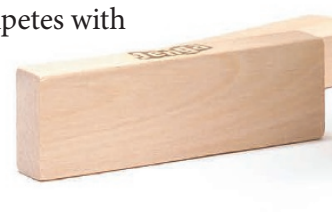

4

daily oral

administration

of H3B-8800

inhibited the

growth of

leukaemia cells

containing mutations in

SF3B 1

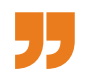

pladienolide for binding to the SF3b complex. In in vitro assays, this molecule, H3B-8800, inhibited both canonical splicing and the aberrant splicing performed by complexes containing cancer-associated mutations.

By contrast, in cell culture and in mouse models, the effects of H3B8800 depended on the mutational status of the spliceosome. In mouse xenografts, daily oral administration of $\mathrm{H} 3 \mathrm{~B}-8800$ inhibited the growth of leukaemia cells containing mutations in SF3B1, but did not substantially affect growth of SF3B1-wild-type cells. Similarly, in patient-derived xenograft (PDX) mouse models using cancer cells derived from individuals with acute myelogenous leukaemia, H3B-8800 reduced growth of the SF3B1-mutant tumour but not the tumour with wild-type SF3B1. Similar results were found in PDX models of chronic myelomonocytic leukaemia. Three PDX models derived from tumours with mutations in SRSF2, which encodes another component of the spliceosome, were sensitive to H3B-8800; three PDX models with wild-type SRSF2 were not.

To understand why H3B-8800 modulates the splicing of both wild-type and mutant spliceosome complexes but only kills spliceosome-mutant cells, the authors examined the transcripts produced in these two situations. H3B-8800 induced exon skipping and intron retention at a similar rate in SF3B1-mutant and wild-type cells. Importantly, H3B8800 also preferentially inhibited the splicing of short, GC-rich introns, even relative to other pladienolide analogues. Genes encoding spliceosome components tend to contain GC-rich introns and are thus affected in cells treated with H3B8800 . As cells carrying spliceosome mutations depend on the wild-type spliceosome for survival, interventions that reduce the quantity of spliceosome factors could tip the balance in favour of cell death in spliceosome-mutant cells but not in wild-type cells.

This study provides a new, orally available modulator of the SF3b splicing complex. H3B-8800 is currently in a phase I trial in patients with splicing factor mutations in leukaemia, and Buonamici is exploring the potential of this compound in combination with other approved and experimental therapies. H3B-8800 could potentially be used in a tissue-agnostic manner to treat tumours with mutations in spliceosome components.

Megan Cully

ORIGINAL ARTICLE Seiler, M. et al. H3B-8800, an orally available small-molecule splicing modulator, induces lethality in spliceosome-mutant cancers. Nat. Med. https://doi.org/10.1038/nm.4493 (2018) 